\title{
Partitioning (Facade) and Identity in the Historical Context Case of Zonouz City
}

\author{
Hassan Khalili Zonouz ${ }^{1}$ \\ ${ }^{1}$ Department of Architectural Theories and Conservation, Faculty of Architecture, Azerbaijan National Academy \\ of Sciences, Baku city, Azerbaijan
}

Correspondence: Hassan Khalili Zonouz, Department of Architectural Theories and Conservation, Faculty of Architecture, Azerbaijan national academy of sciences AZ1073, 115, H. Javid ave., Baku city, Azerbaijan. Tel: 99-45-1456-8928; 98-91-2350-8636. E-mail: hassan.khalili@parasmoon.com; hassankh170@gmail.com

Received: March 1, 2018

doi:10.5539/jsd.v11n6p70
Accepted: June 26, $2018 \quad$ Online Published: November 29, 2018

URL: https://doi.org/10.5539/jsd.v11n6p70

\begin{abstract}
City, identity, and urban landscape are interconnected with each other. Urban restoration is the final objective of the study and implementation of partitioning in historical contexts. The identity of city and its determining urban elements were always discussed while the urban passages are the most prominent elements in creating the historical context of urban landscape. The present study aimed at investigating the implementation of favorable partitions by considering vitality in historical contexts. This study analyzed the identity, originality, and partitioning to apply the principles defining a logical solution and creating the sense of belonging, collective memories, sense of place, and points related to environmental psychology in the historical contexts. Using some landscape architecture principles by considering some parameters such as originality, identity, and social-cultural elements can contribute to idealizing the identity partitioning to historical contexts and its adjacent points in urban areas. As a result, the identity concepts and parameters can interpret the partitions in terms of thought, design, and implementation of the principles used in the architecture.
\end{abstract}

Keywords: identity, originality, urban landscape, partitioning, the principle of façade design in historical context, Zonouz, East Azerbaijan

\section{Introduction}

Partition walls are the result of the skeletal structure of public spaces and are considered as the full space of architecture. Public spaces as square, street, and passage are the empty spaces belonging to the urban residents. Empty spaces are used for transportation, transit, travel, and other citizenship affairs. The partition structure of all passages is linear, which separates the private ownership of urban units from collective spaces. This structure is affected by urban planning, natural geography, the elements involved in geographical context, social culture, and other historical experiences. The architectural culture of each region plays a role in determining form, decorative and artistic elements, available materials, governing the economic status, and attitude of urban activists in their creation.

Partition is a scene for implementing the visual landscape of the city ordering the effective elements such as the horizon, rhythm, coherence, and other indicators including the landscape indicators. In addition, it is required in the city as the residential, commercial, office, and educational units. Based on this definition, partition is not considered as a physical element but a psychological, artistic, and social element in the city. Such an attitude requires a serious attention to functional materials in creating the dimensions, form, and context of designs. In addition, it highlights the concept and meaning than function, form, and environment and considers the artistic place and other values to be superior. These subjects make the designer pay more attention to each principle and observe the scientific and philosophical knowledge because there is a mutual relationship between city and citizen and each element on the partition has a psychological and bio effect on the behavior and spirit of citizens.

Environment is the physical settlement of human (Gifford, 1997) and partition is a tangible part of urban environment, which indicates the environmental effects in the citizen's mind and behavior. For this reason, environment is considered as a set of stimuli depending on the responses presented by the organism. The readability of urban landscape is to easily recognize the urban components and associate them in an interconnected form in the mind (Lynch, 1392). Façade is one of the most important elements in street playing a 
significant role in its readability. Façade promotes the quality of its surrounding space in terms of character, landscape, readability, aesthetics, and the like, which is directly related to psychological sustainability. We affect and are affected by the environment and should observe and understand the environment to create such a mutual relationship. It is possible by seeing, hearing, smelling, and touching the environmental stimuli (Carmona, 2010). Façade is like a dress displaying the skeletal and visual character of a building. Façade should be coordinated with the bodies forming the totality of a street. Meanwhile, each building should display the dominant pattern of facades to create a mental image of the environment and encourage the citizens to attend the designed space. A set of facades form a totality called "urban landscape", and the collective memories of citizens are formed due to attending in the urban spaces (Tavassoli, 2000).

In general, partitions have a main role in the values of urban landscape and determine its identity. Creating the sense of place, sense of belonging, and memory are the features of partitioning and should be studied due to the role of environmental psychology, sociology, and culture.

In the present study, Zonouz in East Azerbaijan was selected as case study, considering its architecture and history added identity and originality to other factors. For this reason, studying and achieving the design idea are needed to achieve the concept of partition design in historical contexts and intact cities in terms of originality and identity. The most important questions raised here are as follows: How can such an idea be achieved? Which solution can provide the necessary relationship between partitioning and identity? How can we manifest vitality and originality?

It seems that the above-mentioned factors should be considered to achieve such an objective. It was assumed that the study sequence of identity, originality, privacies in historical contexts, environmental psychology, culture and cultural elements, principles of landscape and partitioning, and finally recognizing the functional materials will contribute to the design and restoration. Based on this case in the present study, analyzing the data and matching the studies to the required partitioning in Zonouz are the most important objectives.

\section{Historical Context}

Considering the historical context as the creating scene of urban region and historical urbanism with all its aspects, its basis will be the empty and full spaces. The full spaces are the buildings and mansions and the empty spaces of urban context are regarded as a set of passages. The passages which are used because of creating the relationship to full spaces and their ownership are allocated to the public both psychologically and spiritually. Historical context is typically the result of historical periods which gradually developed into its final and valuable form. The buildings and partitions with architectural and artistic parameters are the results of different historical periods. The architects of each society used their art and knowledge for its development and for this reason, there is a building with a history of tens of years or centuries next to another modern building. Thus, the historical context is the result of artistic and social development in the society including the social, political, economic, as well as the life experiences.

City is always in the process of continuous transformation. Urban form is a certain period which can be considered as a temporary situation between the past and future. Thus, different interpretations of city are obtained by considering it as a living creature (Francois \& Gulgonen, 1997). The reason for not observing the difference in a historical context in terms of structure and materials is that the technical evolutions and artistic methods followed the slow changes in the historical curse. Studying the historical context of Zonouz and the historical usage of rubble trench in buildings and creating the empty and full spaces showed a similar implementation for a few-hundred-year history (Figure 1). Although historical differences can be observed in the historical context, distinguishing it needs accuracy and recognition due to the difference in the used elements. 


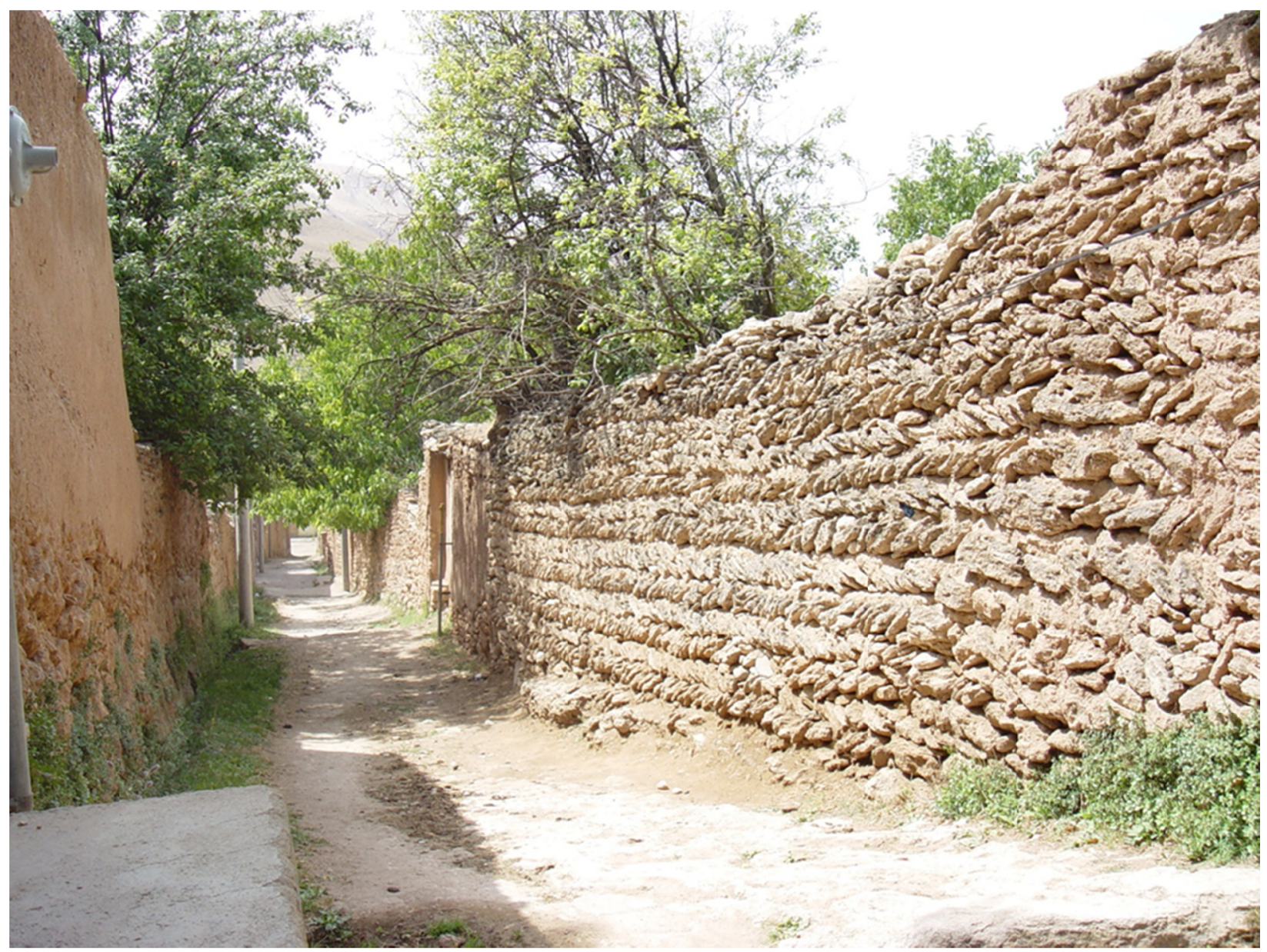

Figure 1. The historical context of Zonouz and the technique of using rubble trench in the partitions facing the passage (Photo by author)

Historical contexts are considered as the inevitable fact of the cities with historical background. They were the member of city in the past including the material and spiritual business and traffic of people at that time. In other words, old urban contexts are creative places for understanding the place of human in the world and its association with the past. They are the places for changing the environment and attitude to the current and future life (Habibi, 2006). Partitioning the historical context in general and Zonouz in particular depends on the rules which should be studied for achieving the ideas and concepts to protect the urban and historical identity and originality of the current parts leading to urban restoration.

\subsection{The Architecture of Zonouz}

In order to present Zonouz as a case study, its history, architecture and urbanism should be explained as follows:

Zonouz is one of the cities in East Azerbaijan located at 38 degrees and 36 min north latitude and 46 degrees and 56 min east longitude. Its ground distance is $28 \mathrm{~km}$ from Marand and $86 \mathrm{~km}$ from Tabriz. Furthermore, the distance of this city is 46 and 105, respectively, from the ground borders of Jolfa (Iran-Azerbaijan) and Nurduz (Iran- Armenia).

The height of its urban range from free water surface varies from $1629 \mathrm{~m}$ (the pieces beside the river) and 1831 $\mathrm{m}$ (next to the water tank above the Kudelar road). Zonouz with Zonuzaq villages are located above the relatively flat bump with a slope descending from the east to the west. It is limited to the Zonouz Chay River from south and seasonal river valley from north. The eastern limit of this city was formed by Zonuzaq fields and rural gardens while in the west part, Zonouz joins the Zonouz Chay River with a sharp slope. The city was developed to the river in a stacked way due to the relatively appropriate slope and insufficient water in the south while its sharp slope and unstable soil prevents its development toward the northern valley.

Zonouz, with some historical sites, has a long history although a few studies have been conducted in this regard. 
According to Ahmad Parviz, the Iranian archeologist, the first observed work of architecture near to this city dates back to the Arax-Kura era. Although no archeological study was conducted on this city, it was probably a settlement for human beings in all historical periods. The first available citation in urban civilization can be attributed to Moghadasi, the Arab traveler, in the $10^{\text {th }}$ century. He considered Zonouz as a city in his book "Ahsan Al-Taqasim Fi Marefat Al-Aqalim". Hamdollah Mostofi, the geographer of the $15^{\text {th }}$ century, in his book entitled "Nozhat Al-Qolub" wrote that Zonouz is a place full of gardens with grain, grapes, and fruits. During the $18^{\text {th }}$ century, Naser al-Din Shah in this second itinerary wrote, "what a village! It is green and located on a mountainous area as high as Alborz but wider. It has many springs and there is no better place in the world. It is a piece of heaven called Zonouz."

Based on the above-mentioned citations, Zonouz has always been a populated place over time and having such a history is impossible without having an identity which is based on culture, language, architecture, and history.

Currently, the formation of units and their establishment as garden as well as residential units as garden house and the combination of this structure with the current topography created a special landscape for Zonouz.

The most important factor of the city linear formation is due to its topography that the slope is mainly to west and southwest. The surface water is easily discharged due to the appropriate slope. The direction of surface water discharge is mainly to the west due to the south slope. The significant point in surface water discharge is the arrival of surface water discharge streams into the residential gardens. This specific system was created due to different springs in the city and using their water traditionally by the residents over time to irrigate the gardens.

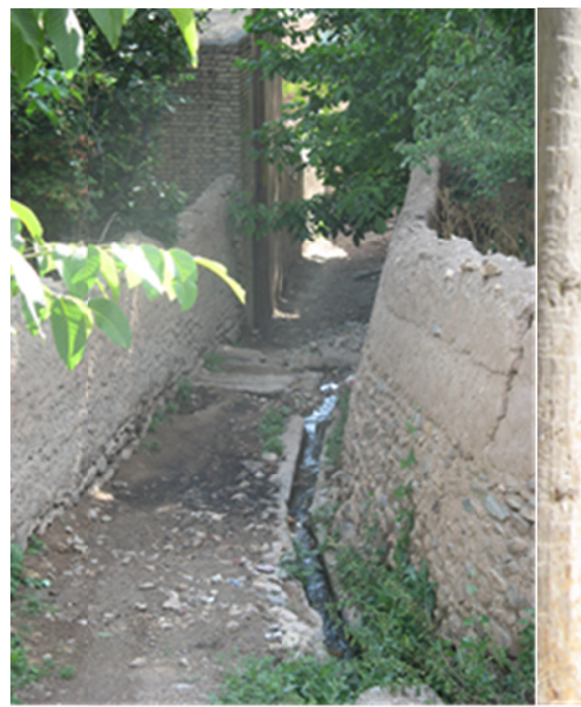

Figures 2 and 3. The effects of natural factors like the passage of passing streams on the formation of passages

(Photos by author)

The access of main streets is mostly as western-eastern. The main core of the city and its historical context is formed by bazaar and Jame mosque and the main passage of the bazaar provided the relationship of quadruple neighborhoods and city exits to Marand and neighboring villages.

The traditional landscape of Zonouz is of great importance in terms of urban landscape. Astonishment is one of the main factors of Zonouz urban landscape whether in terms of visual differences with the similar cities or the context of urban context which is felt by opening the formal spaces towards the unexpected spaces.

The sense of space penetration in the enclosed gardens and their skeletal-visual connection to each other are regarded as other visual features of Zonouz traditional texture which cannot be observed in its modern streets. 


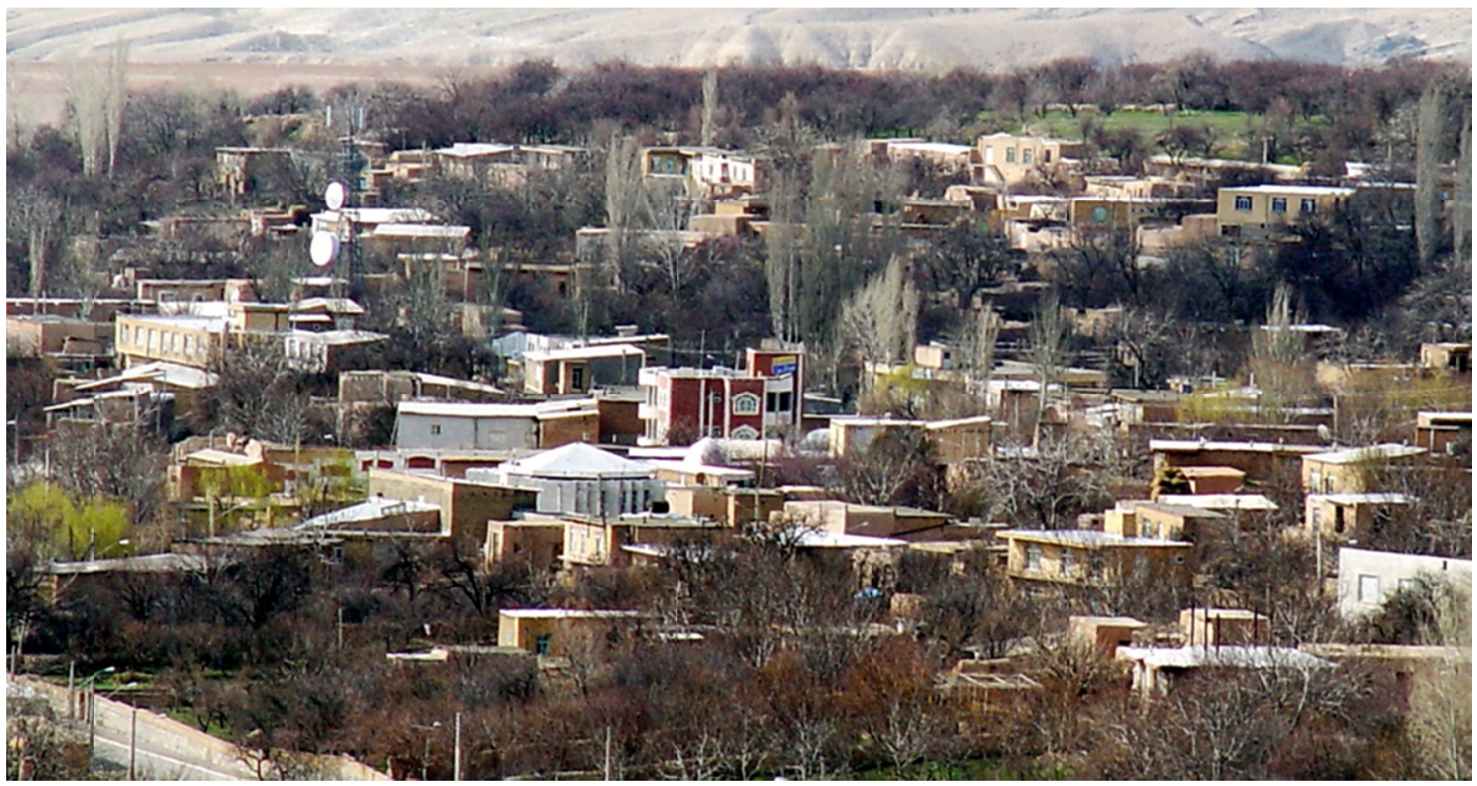

Figure 4. The prospect of Zonouz (The alienation of modern buildings with traditional buildings and landscape destruction is obvious) Photo by Author)

The natural materials and colors used in buildings' façade, the controlled light emitting through the foliage, and even the audio attractions due to the wind passing among the trees in alleyways can be added to the quality of urban environment in Zonouz.

Except the alleyways of the traditional context mentioned in part A, other public spaces of Zonouz included modern streets and squares. The streets were constructed among the historical context and a new elements with old architecture or a few examples of specific rubble trench in Zonouz can be gradually observed over time (Figure 5). It has no visual attraction. The modern squares of Zonouz, except the central square, have the vitality value due to their location in their specific place in the context center. Other squares with scattered spaces without limitation act merely for presentation in the urban environment.

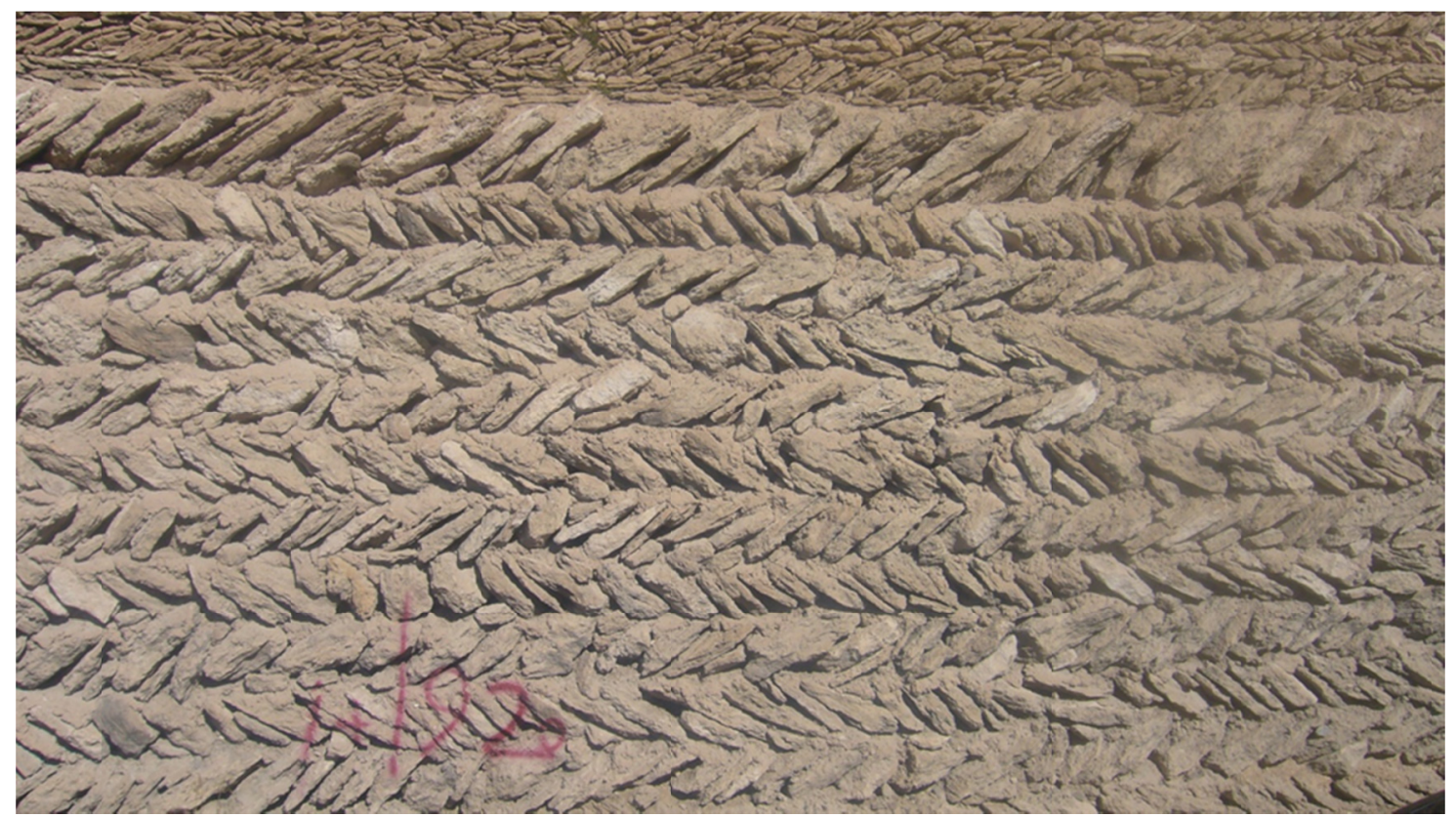

Figure 5. An example of specific dry stone in Zonouz widely used in the facades facing the historical passages (photo by Author) 
The residential spaces of Zonouz, mainly the living spaces, encountered many changes in traditional and modern frameworks since the last decades. The nature of the region, the specific climate of Zonouz, and the potentials of this region played a significant role in forming the unique types of residential space architecture, as well as the complicated structures.

These factors led to a spectacular diversity in the mountainous and moderate nature of the region and its specific rock site for the residential units. The residential pattern governing the region is due to the close relationships between the main activities of the inhabitants and the requirements of this convergence. The gardens full of apple and apricot, agricultural fields, supplying the daily needs, and combining the nature and life style made the builders of residential spaces and buildings consider their houses as a small example of romantic gardens, strengthen their gardening culture, and introduce them to the viewers.

The specific lifestyle of people based on their better status than other gardeners and farmers in the region, their tendency towards establishing beautiful and comfortable buildings, based on the appropriate principles of architecture gradually converted the residential architecture of this region into garden-villa or house, and residential gardens (Khalili Zonouz, 2015)

\subsection{Identity}

In general, identity in historical buildings and contexts can be defined as follows: any skeleton which is created based on the existential philosophy of the work including cultural, scientific, experimental, temporal, and place parameters can be considered as identity.

Identity is a phenomenon created in the historical context of a society. This sense of identity is internalized in the person overtime and integrates the behavior of person and finally the society. The behavioral unity created in the society searches the skeleton to satisfy the behavioral and psychological needs of person and society (Movahed, 2012). Due to the creation of the collective memories of citizens, their sense of belonging and dependency is supplied guiding the citizens towards citizenship with a more active range of settlements. Although the urban identity is the cause of culture of citizens, the process of citizenship is affected which can develop the criteria related to participation and judgment by the viewers and residents (Movaseghi, 2006).

Despite the unique value and place of old textures in the spatial and functional structure of the city, its potentials and strengths created some problems and limitations such as the inconsistency between the skeleton and activity, the presence of urban inconsistent elements, low per capita in some users, lack of appropriate hierarchy in the communication network and lack of penetration to the organic texture in incompatible uses, the presence of defenseless and left spaces, shortage of appropriate public spaces, and the like reduced the significance and value of old texture leading to population displacement and removal of the groups with high financial power (Keshavarz, 2010).

Place identity is the most fundamental concept related to the epistemic aspect of attachment to place. Psychologists believe that the people communicate with a place when that place helps them know who they are. For this reason, the term "identity of place", when used by psychologists, refers to a characteristic of human. Place identity refers to the conscious and unconscious patterns of ideas, feelings, beliefs, attitudes, values, behavioral tendencies, and skills relating the identity of person to the identity of a place and creating some tendencies for making the future relations to the place (Feldman, 1990).

Since the historical evidence is based on originality, its originality will be exposed to risk if the temporal continuity of the work loses its importance due to multiplication (Benjamin, 1979). In other words, the excessive repetition reduces the originality and value of the work. Form is often considered as the most important feature of context originality explaining the mechanism of skeletal forms and elements such as line and surface to develop and determine the nature of space (Bahreini, 1998).

Unlimited similarity is one of the features of modern cities indicating how the modern city lost all its originality and grew based on similarity and imitation (Afrasiabi, 2008). In traditional cities, originality belongs to the city and individuals are a component in the city referring to the individual characteristic. City has a main identity and life becomes meaningless without considering this factor. In this case, context finds meaning and desirability (Habibi, 2001). Thus, the final objective of originality is to implement the final form in the context which is a dynamic reality. Culture affects architecture which is the governing value system. On the other hand, culture is indirectly one of the main bases of psychological life of human beings. The perception of human from the surroundings depends on his culture. Every culture reflects the value system of a social system. In general, knowledge and art are the symbols of this reflection. While knowledge steps towards a logical direction, art deals with the perception of feelings. Architecture, as a social phenomenon, is resulted from culture and is a reflection 
of human thoughts in relation to space, aesthetics, and culture. For this reason, the architectural style of each period is regarded as the reflection of culture and art. Every new style of architecture is based on principles, methods, and traditions of previous styles (Diba, 1999). Thus, urban identity is finding the proportion in the urbanism and architecture system with cultural, social, and economic conditions of citizens. Accordingly, paying attention to social identity (cultural- political) in line with recognizing the real identity of city, historical context, and its partitioning is inevitable. Urban identity is raised as a criterion for developing and factor for promoting the environment quality to provide the fields of participation and security.

\section{Partitioning}

Keeping the current historical context and improving the visual landscape in other parts of the city are the most important objectives of any design. The identity of new parts in historical cities is a function of their partitioning.

Todays, the urban spaces are limited by the bodies in which the form value of enclosing facades is ignored. This challenge reduced the attraction of activities to some extent and the passing space works as a 2-dimensional band for the traffic of vehicles. This visual disturbance, with other visual pollutions, caused some problems with aesthetics in the urban space (Jorg Kurt, 1987). The presence of appropriate forms and patterns in urban partitions can play a significant role in promoting the spatial quality. Each building should show the dominant pattern of buildings and their coordination to create a mental image from the environment and encourage the citizens to attend the designed space. The collection of building façade form a totality called "urban landscape" and the collective memories of citizens are formed due to their presence in urban spaces (Tavassoli, 2000). Such partitions in the historical context are regarded as a considerable issue because the historical context and its aesthetics can be affected by the adjacent and modern spaces. Integrated management thought is in addressing the urban totality, historical areas, facades, and walls in historical passages. The most important factors in the passageology of historical contexts are the visual damages including the vision from inside the context to outside and vice versa depending on the adjacent contexts. For this reason, considering the historical context alone is not sufficient and urban totality or at least the adjacency of historical context should be considered.

Todays, partitioning is conducted in most parts of countries looking for the visual quality promotion. The measures taking place physically on the surfaces are facing the passage such as the extensive partitioning in Baku and the historical part of Tehran.

The space of streets has a linear content and passing function. Partition can represent different values because it is under dynamic conditions and its skeletal elements are integrated with the horizon. Such differences in the areas of values and abilities are more observed in the curve of passage and its intersection showing different landscape values of the city.

Partition and horizon are regarded as the skeletal elements. Horizon is the upper limit of buildings and wall of buildings with different uses. Baseline and combination line are the components of horizon. Baseline can be considered as the upper part of vertical partition and combination line is the final limit of indented partition or visible roof in the sky background.

In general, partition determines the passing space and gives it an environmental concept. The considered elements in partitioning refer to background, rhythm, alignment, proportions, meander, corner, and sign.

Passages in the historical parts of cities are usually the context-oriented and conservative architecture with restoration features reflecting the identity and elements of architecture leading to an emphasis and dynamism of its features. The architecture of historical passages is a part of whole, which regards the coherence of collective values and observes the context in partition to generalize the content to the adjacent buildings. Technologically, the partitions in historical contexts have an appropriate height to observe the horizon because with the current technologies, the maximum of two-floor buildings could be executed. For this reason, the created skeletal order plays a significant effect on creating the sky. Rhythm in piers and facades of historical context indicate the proportion and order, which is considered as the significant element of partition and landscape so that the rhythm of piers is sometimes reflected in the horizon. The establishment, proportion, and details of window are related to the observation of aesthetics and cultural principles as a part of architectural identity in the historical context of cities.

Environmental psychologist believe that strengthening the emotional links with place links with place plays a significant role in overcoming the crisis of identity in the present era giving the sense of sustainability and immortality to human in this changing world (Hay, 1998). Environmental psychology focuses on the emotional interaction between human and place as sense of place to strengthen the sense of belonging, attachment to space, security, identity, and originality in human (Falahat, 2006). 
The architect who wants to restore the previous created space should know the effect of place and time which should be used in this created space.

Every place we are living has a form which can be identified by the qualitative aspect of geometry. Place is where objects are located including both qualitative and quantitative aspects. Interestingly, the qualitative aspect is more compatible with human spirit (Tahbaz, 2004). Accordingly, the qualitative aspect restoring the passage partition which is directly related to most residents and viewers of historical areas requires paying attention to the principles which can provide the emotional and logical relationship between human and environment. Although the principles and elements like background, alignment, meander, corner, sign, proportion, and rhythm were considered by active architects in historical contexts in different studies, it seems that the principles of contemporary landscape are more approximate to the objectives of partitioning. Such principles are sometimes compatible with previous elements but are different in some other elements such as balance, simplicity, focalization, rhythm and line, scale and proportion.

A balanced combination is created if the visual energy of all elements is organized in such a way that no part can destroy the visual energy of other parts (Saremi, 1997). Balance in architecture leading to peace is possible in a balanced or unbalanced way. Simplicity is an existence with beautiful unity removing unconformity and meaningless factors (Farhoudi, 1999) leading to intimacy and an strengthened identity in architecture. In addition, it can cause better reliability of decorations and understanding the limits of historical and vertical lines, destroys the contradiction of elements, and provides the possibility of repetition. The third principle is focalization related to the determining factors in the historical context and passages on which partitioning is conducted. In the focal center, the elements are not contrary to each other and should be in such a way that the elements inside the focal center are considered. This focal point can be an excellent building, historical façade, simple design with focalization, lighting on specific decoration or any other form of architectural elements in which the environment can give it focalization and easily transfer the concept of historical environment. The fourth principle is rhythm and line and the simplest form of repetition is the linear type including many components (Filinejad, 2006). However, the components should not be necessarily similar to each other for considering by repetition method (Falamaki, 2008). They may have a common feature or a common denominator while they are unique belonging to a family. Based on these physical features, the architectural forms and spaces can be organized by repetition. Rhythm can be considered as the supplementary of frame and balance in an artistic work. If an architect pays attention to rhythm and balance, he can present some considerable examples of conscious compositions. Line leads to pursuing the decorative and partitions by eyes and mild curves cause more beauty and create the necessary cohesion. The fifth principle is proportion and scale. The most functional and important of these proportions and scales is the visual and conceptual scale. In fact, scale creates the image and perception of viewers from a subject like an architectural building. The scale resulted from the perception of people in a society from the dimension and proportions of spacer and surrounding images is defined by every person from his childhood in his mind (Bolkhazi, 2009). Proportions are a determining factor for coordination and lack of proportion causes irregularity. Proportion was used in classic to modern works by the deconstructionists of the contemporary era. They all sought to express the aesthetic proportions of their era (Rezazade, 2007).

Proportion in architecture in terms of aesthetics is the quantitative comparison of dimensions and sizes in architectural works. Thus, the architect uses the aesthetic proportions in aesthetics for the manifestation of balance. According to Mitean, explaining the aesthetics in relative and its proportion is as supplying the proportions of architect to temporal and place conditions.

The mentioned quintet principles are not sufficient to restore the identity to historical contexts and guarantee the restoration because it needs another form to include identity and should blow the soul of identity from scientific, managerial, and executive perspectives. The research project for implementing the above-mentioned quintet principles had two knowledge layers called "conceptual and technologic parameters". Conceptual layer refers to the elements affecting the form, background, symbol, and any sign related to culture, traditions, social values, beliefs, and nationality of residents. Culture can express many parameters of social and identity groups such as social behavior, speech, manner, art, and economy (Kasmayi, 2009). It is regarded as a set of factors which can create better life based on time and place factors regardless of discriminations and contradictions in the society. Thus, in culture, time and evolution are linked and whatever used for the current generations is called "cultural heritage" (Memarian, 2005). Each society has its own specific culture forming its architecture basis. The architecture of that society is the objective image of that culture and the culture of each society responds to the formation of spaces (Falamaki, 2001).

As for technology, there are two simultaneous items. The first time is the materials of architecture used in terms of architectural techniques. Such materials are traditionally related to climate and are used with the technological 
and artistic knowledge of any climate by the architects of that society to create the spaces.

Materials, like some tools in partitioning, provide us with additional abilities in architecture. Design in open space and materials such as light, shadow, and water can strengthen such possibility.

There is a dual nature inside any material which should be understood by designers. Each material should be used based on its technical and structural proportions. If we cannot understand the limitations and capabilities of materials, creating the partitions with the expected features is impossible (Vafamehr, 2014). On the other hand, a design in line with the natural beauty and spirit of materials is required to have success in implementation. These materials were used by previous architects in creative and reliable methods and were interwoven with the spirit of people in terms of repeating and transferring knowledge from a generation to another one.

In such cases, the better proportions of modern materials or weaknesses of traditional techniques and materials are raised. The use of reinforcing materials in partitioning to remove the structural and environmental deficits of techniques and materials in the partitions of historical context is allowed unless it can damage the identity. Todays, the materials such as Methacrylate resins are used in the world to increase the capabilities of some projects are based on the sustainable architecture of partitions in restoring the historical contexts.

\section{Case Study}

Zonouz was introduced as the case study in this project. Zonouz is a city with social people The city has a very old civilization (Riyazi, 2008).and is similar to other cities of Azerbaijan like Ordubad and Khinaliq in Azerbaijan country and is similar to Iqdir and Mudurnu in Anatolia and the cities of Tabriz and Ahar in the East Azerbaijan in terms of architectural style. However, it has some unique features with some similarities with other cities having the same civilization and some differences in terms of skeleton, landscape, and completely natural materials. This city has a complete sustainability with its nature because the materials such as rubble trench and soil and mud mortar are removed as raw from its environment, without causing any damage to the nature. Another main difference is related to the planning type of garden houses with very low density in terms of occupation level.

There is spring water in the alleys of this city and no alley can be found without a stream. Soft elements such as streams, shadow of trees, and sunlight emitting though the foliage and partitions to the alleys are perceived well.

The techniques of partitioning and dry stone in alleyways and fence of garden houses are unique in this city giving a different identity from the adjacent cities as if its formation has been inspired by the girls' braided hair in the form of Horuk. For this reason, the privacies of alleys in the historical context should be determined as the size of the current passage width to guarantee the presence of soft elements.

The most appropriate option to restore the historical context is protecting the historical context left from the previous construction projects (Figure 6) and the next step is organizing the urban landscape. Organization can bring the necessary coherence in the historical context and create the logical relationship providing its urban identity (Figure 7).

Figures 1, 2,3, and 5 illustrate some examples of partitioning in the historical context and alleyways. As shown, most identity and psychological needs and principles were traditionally observed in its partitions, which supplied the viewers' peace and restore the collective memories. These façades include the originality of climatic architecture and include the necessary intimacy for citizens and tourists. The relationship between the historical contexts and adjacent areas can be established by observing the principles of partitioning and identity issues. 


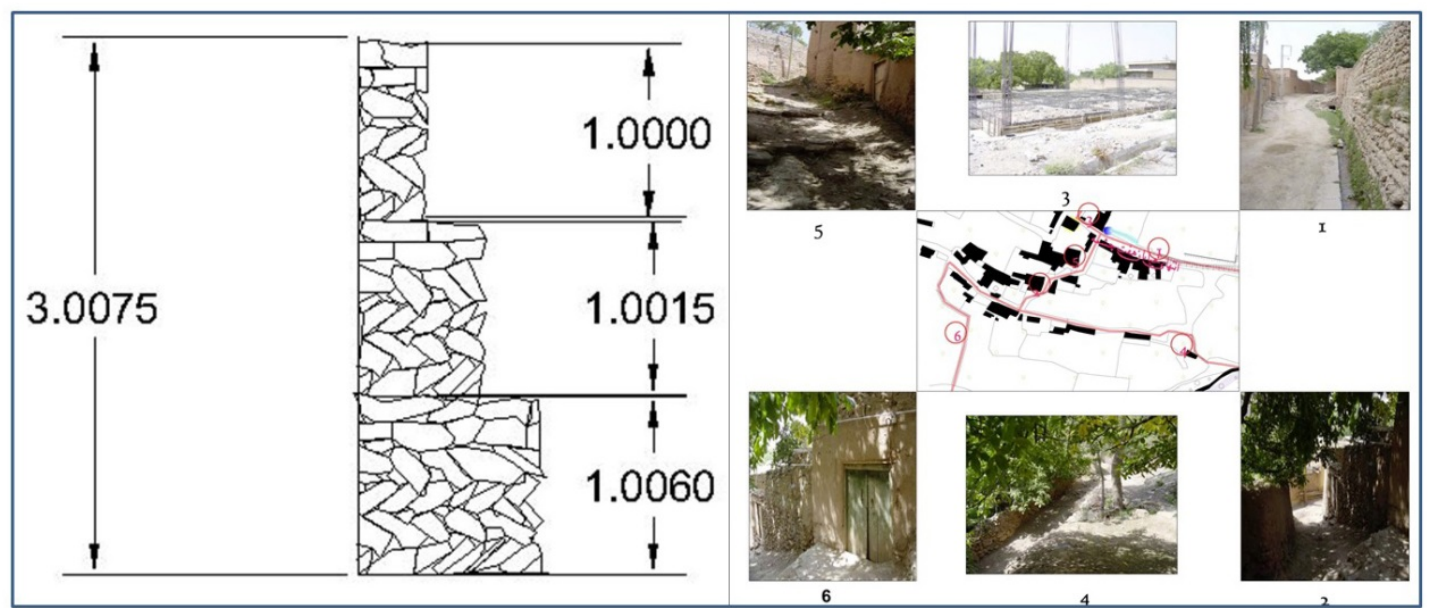

Figure 6. The proposed details for partitioning in the by-lanes of the historical context and alleyways (Building facade in these parts was considered as the current examples) (Created by Author)
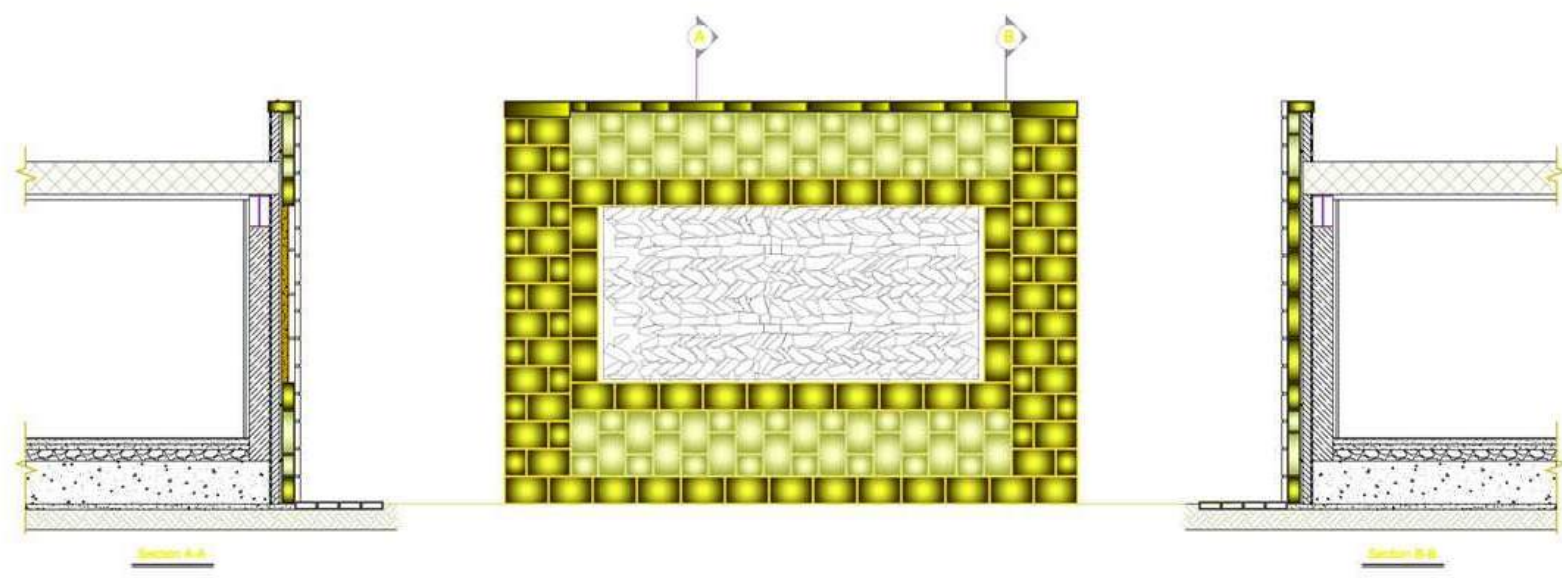

Figure 7. The proposed details for the adjacencies with alleyways and high-populated areas in the historical context (This partitioning is a combination of Hurak, Rikin and column, the whole work is a combination of local wall installation and can provide the required principles as repetition next to each other. On the other hand, it has the cultural urban parameters of Zonouz) (Created by Author)

\section{Conclusion}

The architecture of passages in historical contexts is context-oriented and conservative architecture includes the restoration features. It is an architecture reflecting the identity and elements of architecture of the surrounding buildings and providing the emphasis and dynamism of these features.

In order to interfere in partitioning historical contexts with identity, the area of thought and planning needs a serious attention in three sections including the philosophical, aesthetic, and technical issues. A successful plans should involve these three components. Philosophical issues define the cases in terms of originality, identity, type of materials and form of façade for implementing the piers because each society has its own beliefs, symbols, myths, and forms with clear definitions. Circle, swastika, or square in a society has different concepts than other societies. The concepts are institutionalized in the people's minds in that society in terms of history forming a part of social culture in that society. The placement of forms and volumes next to each other with mathematical and visual proportions along the type of light and color can make these forms and volumes desirable to the viewers in order to enjoy watching. This aspect depends on environmental psychology and human taste and can be a definition of aesthetics. When urban aesthetics is raised, considering different collective tastes, peace, empty and full spaces, pause and movement, sense of belonging, collective memories, 
vision from outside to inside, and vice versa are affected by aesthetics. Aesthetics in historical contexts depends on the order rate manifested in partitions. The type and quality of materials in implementing the forms of partitioning and its intimacy to urban residents affect and are affected by the philosophical and aesthetic points of partitioning. Different societies used the nature and their capabilities in applying the materials based on their social economy and recognition for historical contexts gradually to make the shell of urban passages desirable. The used technique was local and mainly based on their needs and objectives. The diversity of materials is more limited and recycled without causing any damage to the nature. It is true at least on Zonouz as the case study.

Each plan has its own specific rules. In case of historical passages, it seems that the landscape architecture principles can be successful in implementation due to the psychological relation of human to the structure of partitions and more attention to aesthetics and five senses in the environment as well as using the more compatible materials. This objective can be achieved by recognizing the thought of identity and originality with traditional elements and materials and their social-cultural manifestation in facades.

\section{Refrences}

Afrasyabi, A. (2008). Khosoosi va omoomi dar taghaboli nasaz. Nama.

Bahreyni, S. H. (2006). Farayand e tarahi shahri. Tehran University Press.

Benjamin, W. (1979). The Work of Art in the Age of Mechanical Reproduction (H. Arendt, edi.). Fontana-Colins, Bolkhazi, H. (2009). Hendeseye Khiyal ve zibayi ( $1^{\text {st }}$ ed.). Asare Honari pres, Tehran.

Carmona, M. (2010). Public Places Urban Spaces (2 ${ }^{\text {nd }}$ ed.). Elsevier Ltd.

Diba, D. (1999). Elham va bardasht az mafahim bonyadi memari Iran, Farhangh ve memari, sal avval, shomare avval.

Falahat, M. S. (2006). Mafhoom e hess e makan va avamel e shekl dahandeye an. Honarhaye ziba mag.

Falamaki, M. (2001). Risheha ve Gharayeshhaye Nazari memari, Faza, Tehran.

Falamaki, M. (2008). Memari va mousighi, Elmi farhanghi Faza, Tehran

Feldman, R. M. (1990). Setllement Identity: Psychology Bonds with Home Places In A Mobile Society. Environment and Behavior. https://doi.org/10.1177/0013916590222002

Fili, N. G. (2006). Hamsouyi jahane memari va musighi, Farhangh va ahangh, sale dovvom, khordad ve tir.

Francois, L., \& Gulgonen, A. (1977). Morphologie urbaine ET typologies architecturale. Paris: Institut d'études ET de 80echerché architecturales et urbaines, Ministère de la culture et de l'environement.

Gifford, M. (1997). Taghyire eghlim, translated by Khaledi, S., Sara publication, Tehran.

Habibi, S. M. (2000). Fazaye Shahri, hayat e vagheyi va khaterehay jamee. Soffeh mag,

Habibi, S. M. (2006). Description of Stream of Though in Contemporary Iranian Architecture and Urbanism, Cultural Research Center, Tehran.

Hay, R. (1998). Sense Of Place In Developmental Context. Environmental Psychology Journal. https://doi.org/10.1006/jevp.1997.0060

Jorg Kurt, G. (1987). A sthetik der Architektur: Grundlagen der Architektur-Wahrnehmung, W. Kohlhamme, Stuttgart.

Kasmayi, M. (2009). Eghlim ve Memari, Baztab publication, Tehran.

Keshavarz, M. et al. (2010). Geological evolution of the concept of reinventing the city as a new approach in urban contexts. Iranian Islamic City Journal, 1, 73-92.

Khalili Zonouz, H. (2015). Effects of climate conditions in formation and development of Zonuz's historical garden city architecture. Journal of Architecture and Urbanism, 39(2).

Lynch, K. (1960). The Image of the City. The MIT Press.

Memarian, G. (2005). Seyri dar mabani Nazariye Memari, Soroushe Danesh, Tehran.

Movahed, A. (2012). Recognition of Physical Identity in Islamic Cities (Case Study: ReyCity). Journal of Regional Planning, 5, 51-37.

$\begin{array}{cccccc}\begin{array}{c}\text { Movasaghi, } \\ \text { https://www.civilica.com/Paper-SCSD01-SCSD01 }\end{array} \text { 029.html } & \text { Ryremlou, } & \text { (2006). } & \text { Retrieved } & \text { from }\end{array}$ 
Rezazade, R. (2007). Memari zamine gara. Khak Publication, Tehran.

Riyazi, J. (2008). Ghozari bar shahre elm va adab «Zonouz». Hour, Tabriz.

Saremi, A., \& Radmehr, T. (1997). Arrzeshhaye paydari dar memari Iran, Sazman Mirase fsarhanghi, Tehran.

Tahbaz, M. (2004). Shekl e moghadas, Soffeh mag, 38.

Tavassoli, M. (2000). Tarrahi fazahaye shahri, markaze motaleat va tahghighat memari ve shahrsazi, Tehran.

Vafamehr, M. (2014). Seyri dar andishehaye memari. Elm ve Sanat university press.

\section{Copyrights}

Copyright for this article is retained by the author(s), with first publication rights granted to the journal.

This is an open-access article distributed under the terms and conditions of the Creative Commons Attribution license (http://creativecommons.org/licenses/by/4.0/). 